Research Article

\title{
Existence and Multiplicity Results for Nonlinear Differential Equations Depending on a Parameter in Semipositone Case
}

\author{
Hailong $\mathrm{Zhu}^{1}$ and Shengjun $\mathrm{Li}^{2}$ \\ ${ }^{1}$ School of Statistics and Applied Mathematics, Anhui University of Finance and Economics, \\ Bengbu 233030, China \\ ${ }^{2}$ College of Information Sciences and Technology, Hainan University, Haikou 570228, China \\ Correspondence should be addressed to Hailong Zhu, mmyddk@163.com
}

Received 27 September 2012; Accepted 13 October 2012

Academic Editor: Jifeng Chu

Copyright (c) 2012 H. Zhu and S. Li. This is an open access article distributed under the Creative Commons Attribution License, which permits unrestricted use, distribution, and reproduction in any medium, provided the original work is properly cited.

The existence and multiplicity of solutions for second-order differential equations with a parameter are discussed in this paper. We are mainly concerned with the semipositone case. The analysis relies on the nonlinear alternative principle of Leray-Schauder and Krasnosel'skii's fixed point theorem in cones.

\section{Introduction}

In this paper, we consider the problem of existence, multiplicity, and nonexistence of positive solutions for the following boundary value problem (BVP):

$$
\begin{gathered}
-\left(a(t) x^{\prime}\right)^{\prime}+b(t) x=\lambda f(t, x), \quad t \in I, \\
x(0)=x(2 \pi), \quad a(0) x^{\prime}(0)=a(2 \pi) x^{\prime}(2 \pi),
\end{gathered}
$$

where $I:=[0,2 \pi], \lambda$ is a positive parameter, $f(t, x) \in \operatorname{Car}\left(I \times \mathbb{R}^{+}, \mathbb{R}\right)$, and $a(t), b(t)$ are realvalued measurable functions defined on $[0,2 \pi]$ and satisfy the following condition:

$$
a(t)>0, \quad b(t) \geq 0, \quad b(t) \not \equiv 0, \quad \int_{0}^{2 \pi} \frac{d t}{a(t)}<\infty, \quad \int_{0}^{2 \pi} b(t) d t<\infty
$$


Here, the symbol $\operatorname{Car}\left(I \times \mathbb{R}^{+}, \mathbb{R}\right)$ denotes the set of functions satisfying the Carathédory conditions on $I \times \mathbb{R}^{+}$; that is,

(i) $f(\cdot, x): I \rightarrow \mathbb{R}$ is Lebesgue integrable for each fixed $x \in \mathbb{R}^{+}$, and

(ii) $f(t, \cdot): \mathbb{R}^{+} \rightarrow \mathbb{R}$ is continuous for a.e. $t \in I$.

Due to a wide range of applications in physics and engineering, second-order boundary value problems have been extensively investigated by numerous researchers in recent years. For a small sample of such work, we refer the reader to [1-18] and the references therein. When $a(t)=1, b(t)=m^{2}, \lambda=1$ of $\left(E_{\lambda}\right)$, in [11, 18], by using Krasnosel'skii's fixed point theorem, the existence and multiplicity of positive solutions are established to the periodic boundary value problem:

$$
\begin{gathered}
-x^{\prime \prime}+m^{2} x=f(t, x), \quad t \in I, \\
x(0)=x(2 \pi), \quad x^{\prime}(0)=x^{\prime}(2 \pi),
\end{gathered}
$$

where $f(t, x) \in \operatorname{Car}\left(I \times \mathbb{R}^{+}, \mathbb{R}^{+}\right)$.

In [8], Graef et al. consider the second-order periodic boundary value problem:

$$
\begin{gathered}
-x^{\prime \prime}+m^{2} x=\lambda g(t) f(x), \quad t \in I, \\
x(0)=x(2 \pi), \quad x^{\prime}(0)=x^{\prime}(2 \pi),
\end{gathered}
$$

where $g: I \rightarrow \mathbb{R}^{+}$is continuous and $f: \mathbb{R}^{+} \rightarrow \mathbb{R}^{+}$is continuous and $f(x)>0$ for $x>0$. Under different combinations of superlinearity and sublinearity of the function $f$, various existence, multiplicity, and nonexistence results for positive solutions are derived in terms of different value of $\lambda$ via Krasnosel'skii's fixed point theorem.

Hao et al. [9] use the Global continuation theorem, fixed point index theory, and approximate method to study the following periodic boundary value problems:

$$
\begin{gathered}
-x^{\prime \prime}+a(t) x=\lambda f(t, x), \quad t \in I, \\
x(0)=x(2 \pi), \quad x^{\prime}(0)=x^{\prime}(2 \pi),
\end{gathered}
$$

where $a \in L^{1}(0,2 \pi)$ and $f(t, x) \in \operatorname{Car}\left(I \times \mathbb{R}^{+}, \mathbb{R}^{+}\right)$.

In [10], by using the fixed point index theory, He et al. study the existence and multiplicity of positive solutions to BVP $\left(E_{\curlywedge}\right)$. Motivated by the above works, we establish the results of existence, multiplicity, and nonexistence of positive solutions for BVP $\left(E_{\curlywedge}\right)$ via Leray-Schauder alternative principle and Krasnosel'skii's fixed point in the semipositone case, that is, $f(t, x)+M>0$ for some $M>0$. Notice that we do not need $f(t, x)>0$ for any $t \in[0,2 \pi]$ and $x>0$, which is an essential condition of $[9,10]$.

The main result of the present paper is summarized as follows.

Theorem 1.1. Assume that

$$
f^{0}:=\lim _{x \rightarrow 0^{+}} \max _{t \in[0,2 \pi]} \frac{f(t, x)}{x}<\infty, \quad f^{\infty}:=\lim _{x \rightarrow+\infty} \max _{t \in[0,2 \pi]} \frac{f(t, x)}{x}<\infty .
$$


Then, there exist $0<\underline{\lambda}<\bar{\lambda}$ such that $\left(E_{\lambda}\right)$ has no positive solution for $\lambda<\underline{\lambda}$ and at least two positive solutions for $\lambda \geq \bar{\lambda}$.

Remark 1.2. The main result above is a generalization of [9, Theorem 1.2] and [10, Theorem 1.2] and some other known results, in which $f^{0}$ and $f^{\infty}$ must be zero, besides $f(t, x)$ is positive.

The remaining part of the paper is organized as follows. Some preliminary results will be given in Section 2. In Section 3, existence results are obtained using a nonlinear alternative of Leray-Schauder and fixed point theorem in cones when $\lambda$ is large enough; the proof of Theorem 1.1 is also given.

\section{Preliminaries and Lemmas}

In this section, we present some preliminary results which will be needed in subsequent sections. Denote by $u(x)$ and $v(x)$ the solutions of the corresponding homogeneous equation:

$$
-\left(a(t) x^{\prime}\right)^{\prime}+b(t) x=0, \quad t \in I,
$$

under the initial conditions

$$
u(0)=1, \quad a(0) u(0)=0, \quad v(0)=0, \quad a(0) v(0)=1 .
$$

Lemma 2.1 (see [2, Theorem 2.4], [10, Lemma 2.1]). Assume that (H1) holds and $h \in C\left(I, \mathbb{R}^{+}\right)$. Then for the solution $x(t)$ of the BVP

$$
\begin{gathered}
-\left(a(t) x^{\prime}\right)^{\prime}+b(t) x=h(t), \quad t \in I, \\
x(0)=x(2 \pi), \quad a(0) x^{\prime}(0)=a(2 \pi) x^{\prime}(2 \pi),
\end{gathered}
$$

the formula

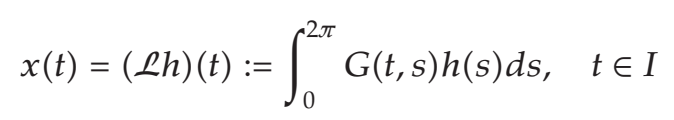

holds, where

$$
\begin{aligned}
G(t, s)= & \frac{v(2 \pi)}{D} u(t) u(s)-\frac{a(2 \pi) u^{\prime}(2 \pi)}{D} v(t) v(s) \\
& + \begin{cases}\frac{a(2 \pi) v^{\prime}(2 \pi)-1}{D} u(t) v(s)-\frac{u(2 \pi)-1}{D} u(s) v(t), & 0 \leq s \leq t \leq 2 \pi, \\
\frac{a(2 \pi) v^{\prime}(2 \pi)-1}{D} u(s) v(t)-\frac{u(2 \pi)-1}{D} u(t) v(s), & 0 \leq t \leq s \leq 2 \pi,\end{cases}
\end{aligned}
$$

and $D=u(2 \pi)+a(2 \pi) v^{\prime}(2 \pi)-1>0$. 
Lemma 2.2 (see [2, Theorem 2.5], [10, Lemma 2.2]). Under condition (H1), the Green's function of the BVP (2.3) is positive, that is, $G(t, s)>0$ for $t, s \in I$.

Remark 2.3. We denote

$$
A=\min _{0 \leq s, t \leq 2 \pi} G(t, s), \quad B=\max _{0 \leq s, t \leq 2 \pi} G(t, s), \quad \sigma=\frac{A}{B} .
$$

Thus, $B>A>0$ and $0<\sigma<1$. In this paper, we use $\omega(t)$ to denote the unique periodic solution of (2.3) with $h(t)=1$, that is, $\omega(t)=(\mathcal{L} 1)(t)$. Obviously, $A \leq\|\omega\|_{\infty} / 2 \pi \leq B$.

Remark 2.4. If $a(t)=1, b(t)=m^{2}>0$, then the Green's function $G(t, s)$ of the boundary value problem (2.3) has the form

$$
G(t, s)=G(|t, s|)= \begin{cases}\frac{\exp (m(t-s))+\exp (m(2 \pi-t+s))}{2 m(\exp (2 m \pi)-1)}, & 0 \leq s \leq t \leq 2 \pi, \\ \frac{\exp (m(s-t))+\exp (m(2 \pi-s+t))}{2 m(\exp (2 m \pi)-1)}, & 0 \leq t \leq s \leq 2 \pi\end{cases}
$$

It is obvious that $G(t, s)>0$ for $0 \leq s, t \leq 2 \pi$, and a direct calculation shows that

$$
A=\frac{e^{m \pi}}{m\left(e^{2 m \pi}-1\right)}, \quad B=\frac{1+e^{2 m \pi}}{2 m\left(e^{2 m \pi}-1\right)}, \quad \sigma=\frac{2 e^{m \pi}}{1+e^{2 m \pi}}<1 .
$$

In the obtention of the second periodic solution of $\left(E_{\curlywedge}\right)$, we need the following wellknown fixed point theorem of compression and expansion of cones [19].

Lemma 2.5 (see Krasnosel'skii [19]). Let $X$ be a Banach space and $K(\subset X)$ a cone. Assume that $\Omega_{1}, \Omega_{2}$ are open subsets of $X$ with $0 \in \Omega_{1}, \bar{\Omega}_{1} \subset \Omega_{2}$, and let

$$
T: K \cap\left(\bar{\Omega}_{2} \backslash \Omega_{1}\right) \longrightarrow K
$$

be a continuous and compact operator such that either

(i) $\|T u\| \geq\|u\|, u \in K \cap \partial \Omega_{1}$ and $\|T u\| \leq\|u\|, u \in K \cap \partial \Omega_{2}$, or

(ii) $\|T u\| \leq\|u\|, u \in K \cap \partial \Omega_{1}$ and $\|T u\| \geq\|u\|, u \in K \cap \partial \Omega_{2}$.

Then $T$ has a fixed point in $K \cap\left(\bar{\Omega}_{2} \backslash \Omega_{1}\right)$. define

In the applications below, we take $X=C[0,2 \pi]$ with the supremum norm $\|\cdot\|$ and

$$
K=\left\{x \in X: x(t) \geq 0 \forall t, \min _{0 \leq t \leq 2 \pi} x(t) \geq \sigma\|x\|\right\}
$$

where $\|x(t)\|=\max _{0 \leq t \leq 2 \pi}|x(t)|$. 

by

One may readily verify that $K$ is a cone in $X$. Finally, we define an operator $T: X \rightarrow K$

$$
(T x)(t)=\int_{0}^{2 \pi} G(t, s) F(s, x(s)) d s,
$$

for $x \in X$ and $t \in[0,2 \pi]$, where $F:[0,2 \pi] \times \mathbb{R} \rightarrow[0, \infty)$ is continuous and $G(t, s)$ is the Green function defined above.

Lemma 2.6 (see [12, Lemmas 2.2, 2.3], [13, Lemma 2.4]). $T$ is well defined and maps $X$ into $K$. Moreover, $T: X \rightarrow K$ is continuous and completely continuous.

\section{Proof of Theorem 1.1}

In this section we establish the existence, multiplicity, and nonexistence of positive solutions to the periodic boundary problem $\left(E_{\lambda}\right)$. The first existence result is based on the following nonlinear alternative of Leray-Schauder, which can be found in [15].

Lemma 3.1. Assume $\Omega$ is a relatively compact subset of a convex set $K$ in a normed space $X$. Let $T: \bar{\Omega} \rightarrow K$ be a compact map with $0 \in \Omega$. Then one of the following two conclusions holds:

(I) $T$ has at least one fixed point in $\bar{\Omega}$.

(II) There exist $x \in \Omega$ and $0<\lambda<1$ such that $x=\lambda T x$.

Since we are mainly interested in the semipositone case, without loss of generality, we may assume that $f(t, x)$ satisfies the following.

(F1) There is a constant $M>0$ such that $f(t, x)+M>0$ for all $(t, x) \in[0,2 \pi] \times(0, \infty)$ and let $F(t, x):=\lambda(f(t, x)+M)>0$. Besides, we introduce the following assumption on $f(t, x)$.

(F2) there exists a continuous, nonnegative function $g(x)$ on $(0, \infty)$ such that

$$
f(t, x) \leq g(x), \quad \forall(t, x) \in[0,2 \pi] \times(0, \infty),
$$

that is,

$$
F(t, x) \leq \lambda(g(x)+M), \quad \forall(t, x) \in[0,2 \pi] \times(0, \infty),
$$

and $g(x)>0$ is nondecreasing in $x \in(0, \infty)$.

Theorem 3.2. Suppose $f(t, x)$ satisfies (F1) and (F2). Suppose further that

(F3) there exists $r>M\|\omega\| / \sigma$ such that

$$
\frac{r}{\lambda(g(r)+M)}>\|\omega\|
$$

where $\sigma$ and $\omega$ are as in Section 2. 
Then $\left(E_{\mathcal{\lambda}}\right)$ has at least one positive periodic solution with $0<\|x+M \omega\|<r$.

Proof. The existence is proved using the Leray-Schauder alternative principle. Consider the following equation:

$$
\begin{gathered}
-\left(a(t) x^{\prime}\right)^{\prime}+b(t) x=\mu F(t, x(t)-M \omega(t)), \quad t \in I, \\
x(0)=x(2 \pi), \quad a(0) x^{\prime}(0)=a(2 \pi) x^{\prime}(2 \pi),
\end{gathered}
$$

where $\mu \in[0,1]$. Problem (3.4) is equivalent to the following fixed point problem in $C[0,2 \pi]$ :

$$
x=\mu T x
$$

where $T$ denotes the operator defined by (2.11), with $F(t, x)$ replaced by $F(t, x-M \omega)$.

We claim that any fixed point $x$ of (3.5) for any $\mu \in[0,1]$ must satisfy $\|x\| \neq r$.

Then we have from condition (F2), for all $t \in I$,

$$
\begin{aligned}
x(t) & =\mu T x(t) \\
& =\mu \int_{0}^{2 \pi} G(t, s) F(s, x(s)-M \omega(s)) d s \\
& \leq \int_{0}^{2 \pi} G(t, s) F(s, x(s)-M \omega(s)) d s \\
& \leq \int_{0}^{2 \pi} G(t, s)(\lambda(g(x-M \omega)+M)) d s \\
& \leq \lambda(g(r)+M)\|\omega\| .
\end{aligned}
$$

Therefore,

$$
r=\|x\| \leq \lambda(g(r)+M)\|\omega\|
$$

This is a contradiction to the condition (F3). From this claim, the nonlinear alternative of Leray-Schauder guarantees that (3.5) (with $\mu=1$ ) has a fixed point, denoted by $\widehat{x}_{1}(t)$, that is,

$$
\begin{gathered}
-\left(a(t) \widehat{x}_{1}^{\prime}\right)^{\prime}+b(t) \widehat{x}_{1}=\lambda\left(f\left(t, \widehat{x}_{1}(t)-M \omega(t)\right)+M\right), \quad t \in I, \\
\widehat{x}_{1}(0)=\widehat{x}_{1}(2 \pi), \quad a(0) \widehat{x}_{1}^{\prime}(0)=a(2 \pi) \widehat{x}_{1}^{\prime}(2 \pi) .
\end{gathered}
$$

Using Lemma 2.5 and condition (F3), for all $t \in I$, we have

$$
\widehat{x}_{1}(t) \geq \sigma\left\|\widehat{x}_{1}\right\|=\sigma r>\sigma \cdot \frac{M\|\omega\|}{\sigma}=M\|\omega\|>0
$$


that is,

$$
\widehat{x}_{1}(t)-M\|\omega\|>0
$$

Let

$$
x_{1}^{*}(t)=\widehat{x}_{1}(t)-M \omega
$$

It is easy to see that $x_{1}^{*}(t)$ is a solution of $\left(E_{\curlywedge}\right)$ which satisfies $0<\left\|x_{1}^{*}+M \omega\right\|<r$. Thus, the proof of Theorem 3.2 is completed.

Theorem 3.3. Suppose that conditions (F1)-(F3) hold. In addition, it is assumed that the following two conditions are satisfied.

(F4) There exists a continuous, nonnegative function $h(x)$ on $(0, \infty)$ such that

$$
f(t, x)+M \geq h(x), \quad \forall(t, x) \in[0,2 \pi] \times(0, \infty),
$$

that is,

$$
F(t, x) \geq \lambda h(x), \quad \forall(t, x) \in[0,2 \pi] \times(0, \infty)
$$

and $h(x)>0$ is nondecreasing in $x \in(0, \infty)$.

(F5) There exists a positive number $R>r$ such that

$$
\frac{R}{\lambda h(\sigma R-M\|\omega\|)} \leq\|\omega\|
$$

Then, besides the periodic solution $x$ constructed in Theorem 3.2, $\left(E_{\lambda}\right)$ has another positive periodic solution $\tilde{x}$ with $r<\|\tilde{x}+M \omega\|<R$.

Proof. As in the proof of Theorem 3.2, we only need to show that (3.8) has a periodic solution with $\widehat{x}_{2} \in C[0,2 \pi]$ with $\widehat{x}_{2}>M \omega$ and $r<\left\|\widehat{x}_{2}\right\|<R$.

Let $X=C[0,2 \pi]$ and $K$ the cone in $X$ in Section 2. Let $\Omega_{1}=B_{r}$ and $\Omega_{2}=B_{R}$ be balls in $X$. The operator $T: K \cap\left(\bar{\Omega}_{2} \backslash \Omega_{1}\right) \rightarrow K$ is defined by $(2.11)$, with $F(t, x)$ replaced by $F(t, x-M \omega)$. Note that any $x \in K \cap\left(\bar{\Omega}_{2} \backslash \Omega_{1}\right)$ satisfies $0<\sigma r \leq x(t) \leq R$, thus $T$ is well defined.

First we have $\|T x\| \leq\|x\|$ for $x \in K \cap \partial \Omega_{1}$. In fact, if $x \in K \cap \partial \Omega_{1}$, then $\|x\|=r$. Now the estimate $\|T x\| \leq r$ can be obtained almost following the same ideas in proving (3.7). We omit the details here. 
Next we show that $\|T x\| \geq\|x\|$ for $x \in K \cap \partial \Omega_{2}$. To see this, let $x \in K \cap \partial \Omega_{2}$, then $\|x\|=R$ and $x \geq \sigma R$; it follows from conditions (F4) and (F5) that, for $0 \geq t \geq 2 \pi$,

$$
\begin{aligned}
T x(t) & =\int_{0}^{2 \pi} G(t, s) F(s, x(s)-M \omega(s)) d s \\
& \geq \int_{0}^{2 \pi} G(t, s)(\lambda(h(x-M \omega))) d s \\
& \geq \lambda h(\sigma R-M\|\omega\|)\|\omega\| \geq R=\|x\| .
\end{aligned}
$$

Now Lemma 2.5 guarantees that $T$ has a fixed point $\widehat{x}_{2} \in K \cap\left(\bar{\Omega}_{2} \backslash \Omega_{1}\right)$, thus $r \leq$ $\left\|\widehat{x}_{2}(t)\right\| \leq R$.

Finally, $x_{2}^{*}(t)=\widehat{x}_{2}(t)-M \omega$ will be the another desired positive periodic solution of $\left(E_{\curlywedge}\right)$. We omit the details because they are much similar to that in the proof of Theorem 3.2.

Now we are in a position to present the proof of Theorem 1.1.

Proof of Theorem 1.1. Consider $v(x)>0$ be an eigenfunction satisfying

$$
\begin{gathered}
-\left(a(t) v^{\prime}\right)^{\prime}+b(t) v=\lambda_{1} v, \quad t \in I, \\
v(0)=v(2 \pi), \quad a(0) v^{\prime}(0)=a(2 \pi) v^{\prime}(2 \pi),
\end{gathered}
$$

corresponding to the principal eigenvalue $\lambda_{1}$. Let $x$ be a positive solution of $\left(E_{\curlywedge}\right)$. Multiplying (3.16) by $x$ and $\left(E_{\curlywedge}\right)$ by $v$, and subtracting we obtain

$$
\int_{0}^{2 \pi}\left(\lambda f(t, x)-\lambda_{1} x\right) v \mathrm{~d} x=0
$$

Since $f^{0}<\infty$ and $f^{\infty}<\infty$, there exist positive numbers $\eta_{1}, \eta_{2}, \epsilon_{1}$, and $\epsilon_{2}$ such that $\epsilon_{1}<\epsilon_{2}$ and

$$
\begin{array}{ll}
|f(t, x)| \leq \eta_{1} x & \text { for } x \in\left[0, \epsilon_{1}\right] \\
|f(t, x)| \leq \eta_{2} x & \text { for } x \in\left[\epsilon_{2}, \infty\right)
\end{array}
$$

with $t \in I$. Let the positive number $\eta_{3}$ be defined by

$$
\eta_{3}=\max \left\{\eta_{1}, \eta_{2}, \max _{\epsilon_{1}<x<\epsilon_{2}}\left\{\left|\frac{f(t, x)}{x}\right|\right\}\right\} .
$$

Then

$$
|f(t, x)| \leq \eta_{3} x \text { for } x \in[0, \infty)
$$


Thus, there exists a $\underline{\lambda}>0$, for $0<\lambda<\underline{\lambda}$ satisfying $\left|\lambda_{1} / \lambda\right|>\eta_{3}$. (3.17) cannot hold, and hence $\left(E_{\lambda}\right)$ has no positive solution for $\lambda<\underline{\lambda}$.

Note that the sublinearity of $f(t, x)$ near $x=\infty$, we can construct a suitable $g(x)$ in (F2) which satisfies $\lim _{r \rightarrow \infty} g(r) / r<\infty$. This means that there exists $\lambda>\bar{\lambda}_{1}$ satisfying (3.3) with $r$ being large enough. There also exists $\lambda>\bar{\lambda}_{2}=R /\|\omega\|(h(\sigma R-M\|\omega\|))$ satisfying (3.14). Let $\bar{\lambda}=\max \left(\bar{\lambda}_{1}, \bar{\lambda}_{2}\right)$. Thus, with the help of Theorems 3.2 and 3.3, $\left(E_{\curlywedge}\right)$ has at least two positive solution for $\lambda>\bar{\lambda}$. This completes the proof of the theorem.

Example 3.4. Let the nonlinearity in $\left(E_{\curlywedge}\right)$ be

$$
f(t, x)=\alpha(t) g(x) \exp \left(-x^{\gamma}\right)
$$

with $\gamma>0, \alpha(t)$ is a continuous function for all $t \in I$ and $g(x)$ is a real coefficient polynomial function which has zero constant term. Then Theorem 1.1 is valid.

Proof. In this case, with the function $f(t, x)=\alpha(t) g(x) \exp \left(-x^{\gamma}\right)$, it is easy to verify

$$
\begin{gathered}
f^{0}:=\lim _{x \rightarrow 0^{+}} \max _{t \in[0,2 \pi]} \frac{f(t, x)}{x}=\lim _{x \rightarrow 0^{+}} \max _{t \in[0,2 \pi]} \frac{\alpha(t) g(x) \exp \left(-x^{\gamma}\right)}{x}<\infty, \\
f^{\infty}:=\lim _{x \rightarrow+\infty} \max _{t \in[0,2 \pi]} \frac{f(t, x)}{x}=\lim _{x \rightarrow+\infty} \max _{t \in[0,2 \pi]} \frac{\alpha(t) g(x) \exp \left(-x^{\gamma}\right)}{x}=0<\infty .
\end{gathered}
$$

Then the conclusion follows from Theorem 1.1 that there exists $0<\underline{\lambda}<\bar{\lambda}$ such that $\left(E_{\lambda}\right)$ has no positive solution for $\lambda<\underline{\lambda}$ and at least two positive solutions for $\bar{\lambda} \geq \bar{\lambda}$.

\section{Acknowledgments}

$\mathrm{H}$. Zhu was supported by the NSF of the Educational Bureau of Anhui province (nos. KJ2012B002, KJ2012B004, and 1208085QA11). S. Li was supported by the NSF of China (no. 11161017).

\section{References}

[1] R. P. Agarwal, D. O’Regan, and B. Yan, "Multiple positive solutions of singular Dirichlet second-order boundary-value problems with derivative dependence," Journal of Dynamical and Control Systems, vol. 15 , no. 1, pp. 1-26, 2009.

[2] F. M. Atici and G. S. Guseinov, "On the existence of positive solutions for nonlinear differential equations with periodic boundary conditions," Journal of Computational and Applied Mathematics, vol. 132, no. 2, pp. 341-356, 2001.

[3] A. Cabada and J. Cid, "Existence and multiplicity of solutions for a periodic Hill's equation with parametric dependence and singularities," Abstract and Applied Analysis, vol. 2011, Article ID 545264, 19 pages, 2011.

[4] A. Cabada and J. J. Nieto, "Extremal solutions of second order nonlinear periodic boundary value problems," Applied Mathematics and Computation, vol. 40, no. 2, pp. 135-145, 1990.

[5] J. Chu, P. J. Torres, and M. Zhang, "Periodic solutions of second order non-autonomous singular dynamical systems," Journal of Differential Equations, vol. 239, no. 1, pp. 196-212, 2007.

[6] J. Chu and M. Li, "Positive periodic solutions of Hill's equations with singular nonlinear perturbations," Nonlinear Analysis: Theory, Methods E Applications, vol. 69, no. 1, pp. 276-286, 2008. 
[7] A. Feichtinger, I. Rachůnková, S. Staněk, and E. Weinmüller, "Periodic BVPs in ODEs with time singularities," Computers E Mathematics with Applications, vol. 62, no. 4, pp. 2058-2070, 2011.

[8] J. R. Graef, L. Kong, and H. Wang, "Existence, multiplicity, and dependence on a parameter for a periodic boundary value problem," Journal of Differential Equations, vol. 245, no. 5, pp. 1185-1197, 2008.

[9] X. Hao, L. Liu, and Y. Wu, "Existence and multiplicity results for nonlinear periodic boundary value problems," Nonlinear Analysis: Theory, Methods \& Applications, vol. 72, no. 9-10, pp. 3635-3642, 2010.

[10] T. He, F. Yang, C. Chen, and S. Peng, "Existence and multiplicity of positive solutions for nonlinear boundary value problems with a parameter," Computers $\mathcal{E}$ Mathematics with Applications, vol. 61, no. 11, pp. 3355-3363, 2011.

[11] D. Jiang, "On the existence of positive solutions to second order periodic BVPs," Acta Mathematica Sinica, vol. 18, pp. 31-35, 1998.

[12] D. Jiang, J. Chu, D. O’Regan, and R. P. Agarwal, “Multiple positive solutions to superlinear periodic boundary value problems with repulsive singular forces," Journal of Mathematical Analysis and Applications, vol. 286, no. 2, pp. 563-576, 2003.

[13] D. Jiang, J. Chu, and M. Zhang, "Multiplicity of positive periodic solutions to superlinear repulsive singular equations," Journal of Differential Equations, vol. 211, no. 2, pp. 282-302, 2005.

[14] R. Ma, J. Xu, and X. Han, "Global bifurcation of positive solutions of a second-order periodic boundary value problem with indefinite weight," Nonlinear Analysis: Theory, Methods \& Applications, vol. 74, no. 10, pp. 3379-3385, 2011.

[15] D. O'Regan, Existence Theory for Nonlinear Ordinary Differential Equations, Kluwer Academic Publishers, Dodrecht, The Netherlands, 1997.

[16] I. Rachůnková, "Existence of two positive solution of a singular nonlinear periodic boundary value problem," Journal of Computational and Applied Mathematics, vol. 113, no. 1-2, pp. 24-34, 2000.

[17] P. J. Torres, "Existence of one-signed periodic solutions of some second-order differential equations via a Krasnoselskii fixed point theorem," Journal of Differential Equations, vol. 190, no. 2, pp. 643-662, 2003.

[18] Z. Zhang and J. Wang, "On existence and multiplicity of positive solutions to periodic boundary value problems for singular nonlinear second order differential equations," Journal of Mathematical Analysis and Applications, vol. 281, no. 1, pp. 99-107, 2003.

[19] M. Krasnosel'skii, Positive Solutions of Operator Equations, P. Noordhoff, Groningen, The Netherlands, 1964. 


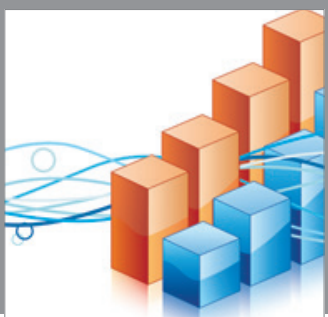

Advances in

Operations Research

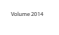

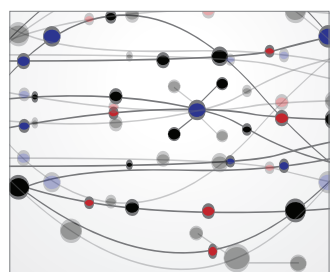

\section{The Scientific} World Journal
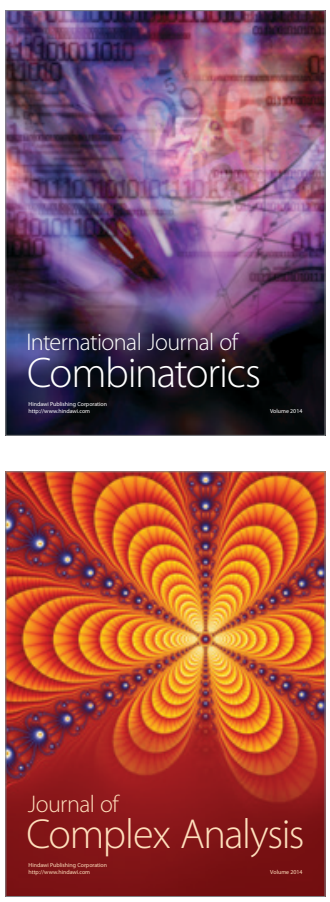

International Journal of

Mathematics and

Mathematical

Sciences
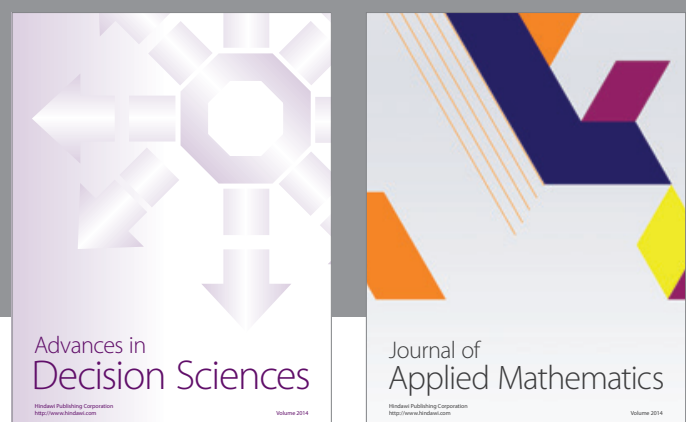

Journal of

Applied Mathematics
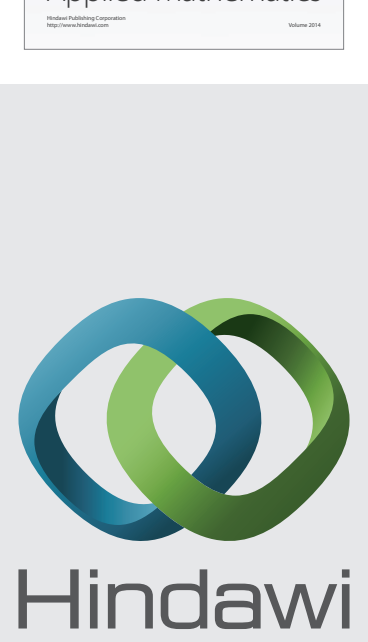

Submit your manuscripts at http://www.hindawi.com
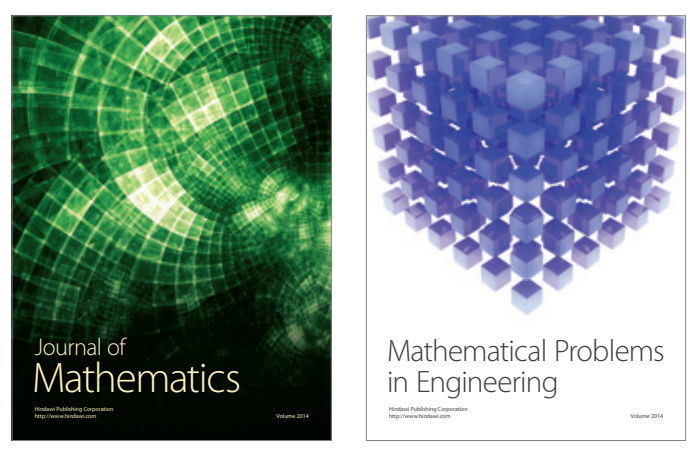

Mathematical Problems in Engineering
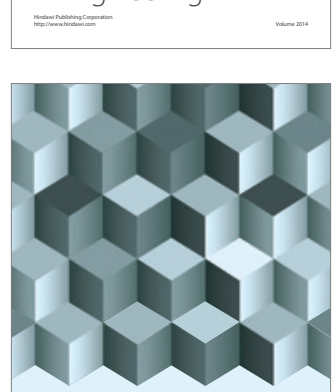

Journal of

Function Spaces
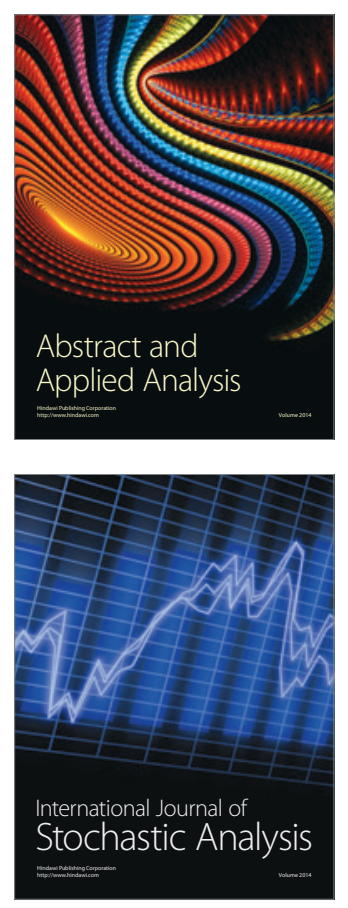

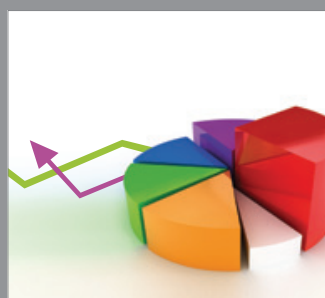

ournal of

Probability and Statistics

Promensencen
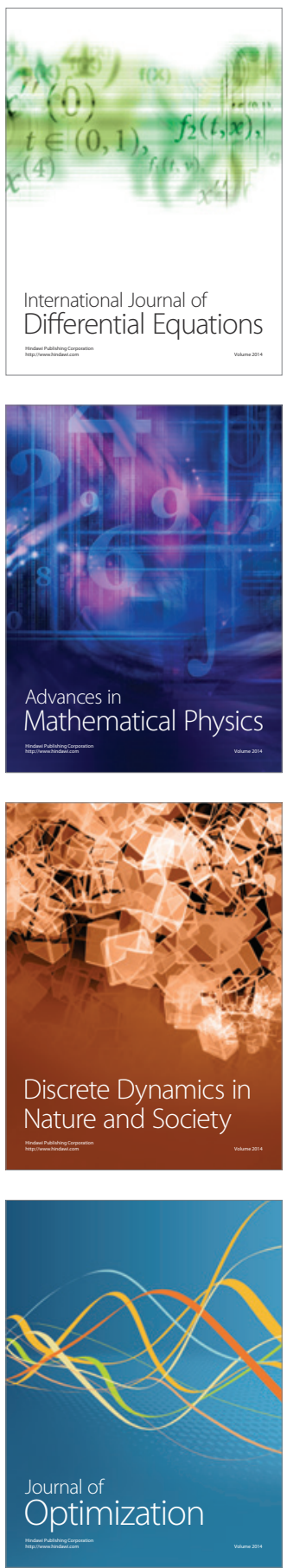